\title{
3D-PRINTED THICK STRUCTURED GELATIN MEMBRANE FOR ENGINEERING OF HETEROGENEOUS TISSUES
}

\author{
Lobat Tayebi ${ }^{1,2}$, Morteza Rasoulianboroujeni ${ }^{2}$, Zhanfeng Cui ${ }^{1}$, Hua $\mathrm{Ye}^{1, *}$ \\ ${ }^{l}$ Institute of Biomedical Engineering, Department of Engineering Science, University of Oxford, Oxford \\ OX1 3PJ, UK \\ ${ }^{2}$ Marquette University School of Dentistry, Milwaukee, WI, 53233, US
}

*Corresponding Author: Email: hua.ye@eng.ox.ac.uk, Phone: +44 (0)1865 617689

\begin{abstract}
Although biological membranes may look like a 2D assembly, they often have complex structures in their 3rd dimension. Using layer-by-layer assembly, 3D-printing can offer an advanced and unique approach for the fabrication of such models. However, printing of some widely used hydrogels, such as gelatin, encounters experimental difficulties due to their rheological properties. In this paper, we (a) discuss the complexities involved in printing gelatin, (b) offer a reproducible approach to overcome such difficulties, and (c) present the detailed design criteria and the production process of such 3D-printed gelatin membranes by exemplifying scaffolds suitable for growth of full-thickness oral mucosa as a heterogeneous tissue.
\end{abstract}

Keywords: 3D-Printing, Gelatin Membrane, Heterogeneous Tissue, Tissue Engineering 


\section{Introduction}

3D-printing has revolutionized research in the area of organ regeneration and tissue engineering through layer-by-layer deposition of biomaterials. However, whilst hard tissue printing has been achieved with various materials, 3D-printing of soft tissue remains a challenge in many cases [13]. Using such a layered assembly, we have produced a thick structured membrane with different porosity and assembly in the different layers/sides for concurrent growth of dissimilar cell types on each side.

Although alginate is known as the major component of hydrogel inks due to its perfect printability and gelation induced by divalent cations [4] it is not a preferred biomaterial for hosting cells due to the relatively poor cell-material interaction [5]. Gelatin is known as a fibroblastattractant and macrophage activator biomaterial, which promotes epithelialization and granulation tissue formation. The final engineered construct can be obtained through either post-fabrication cell seeding or cell encapsulation strategies producing cell-laden prototyped scaffolds [6-8]. The latter could be achieved using gelatin methacrylamide [6] or enzymatic protein crosslinking [7]. Either strategy used, the temperature-dependent sol-gel transition of gelatin plays a critical role in printing procedure. Challenging printing procedure of gelatin-based inks due to premature gelation of this biopolymer, may result in low quality parts [9]. While gelatin could be blended with other polymers $[8,10]$ to improve printability, for pure gelatin/modified gelatin, performing adaptations on the 3Dprinter has been suggested to ameliorate the processing of gelatin hydrogels [6]. However, such strategy might lead to extra cost and might not be applicable to every device.

This paper presents the detailed method of printing gelatin as well as the specific design criteria used for producing the membranes suitable for engineering of heterogeneous tissues. The rheological properties of gelatin have been examined. The present study is expected to increase the 
fundamental understanding of the effect of rheological parameters on the 3D printing procedure of gelatin. The outcome of optimization is a 3D-printed construct with potential applications in tissue engineering of different membrane-shaped organs such as oral mucosa, skin and eye conjunctiva.

\section{Materials and methods}

Gelatin (Type A, from porcine skin, Bioreagent grade) was purchased from Sigma-Aldrich, (St. Louis, MO, USA). 1-Ethyl-3-(3-dimethylaminopropyl)carbodiimide (EDC) and NHydroxysuccinimide (NHS) were obtained from Alfa Aesar (Haverhill, MA, USA)

Envisiontec 3D-Bioplotter ${ }^{\circledR}$ (Manufacturer Series, Germany) was used for the 3D-printing of scaffolds.

The low magnification images of the scaffolds were taken using a Dino-lite Digital Camera. The high magnification imaging of the scaffolds was performed using a JEOL-JSM-6510 Scanning Electron Microscope (SEM, Japan). The samples were dehydrated by immersing in 50, 70 and $90 \% \mathrm{v} / \mathrm{v}$ ascending ethanol series for 20 minutes each. Then they were air-dried overnight, and sputter coated with gold before SEM imaging. Rheological properties of the ink were measured using a shear rheometer (Kinexus, Malvern, UK).

To print any material, seven parameters had to be systematically adjusted: 1) Formulation and rheology of the ink, 2) Material container temperature, 3) Platform temperatures, 4) Dispensing needle diameter, 5) Pressure, 6) Printing speed, and 7) Pre- and post-flow delays.

In our experiment, a 15\% (w/v) solution of gelatin in deionized (DI) water was prepared for printing. Gelatin solution was printed at a material container temperature of $37^{\circ} \mathrm{C}$ and a platform

temperature of $22^{\circ} \mathrm{C}$ using a $250 \mu \mathrm{m}$-diameter needle (25G). Nordson EFD Optimum ${ }^{\circledR}$ general purpose disposable stainless steel blunt end dispensing tips (1/2 inch) were used in this study. 
Printing pressure and speed were adjusted to 0.6 bar and $15 \mathrm{~mm} / \mathrm{s}$, respectively. Pre- and post-flow delays were zero. The reasons for selecting the above printing parameters are described in the Results and Discussion section. The ink was solidified on the platform through sol-gel transition.

The scaffolds were cross-linked by soaking in a $12 \mathrm{mg} / \mathrm{ml} \mathrm{EDC} \mathrm{and} 1.5 \mathrm{mg} / \mathrm{ml} \mathrm{NHS} \mathrm{in} 90 \%$ ethanol solution for 4 hours. To remove the toxic cross-linking agent, the scaffolds were soaked in one liter of DI water which was replaced every hour for 3 hours and before finally rinsing with DI water. After dehydration using 50, 70 and $90 \% \mathrm{v} / \mathrm{v}$ ethanol, the scaffolds were stored in $90 \% \mathrm{v} / \mathrm{v}$ ethanol in a $-20^{\circ} \mathrm{C}$ freezer until used when they were rehydrated and washed carefully as described above.

The printing process and macroscopic view of the printed membrane can be seen in Fig 1 .

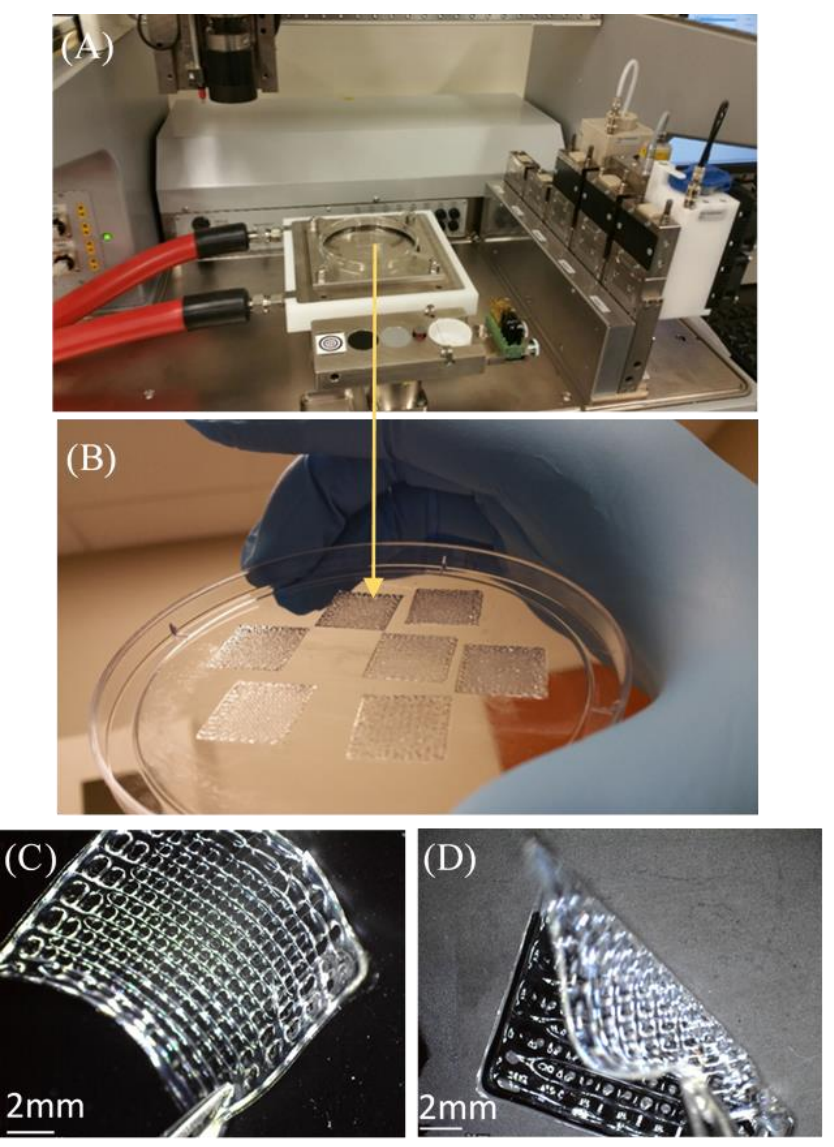

Figure 1: 3D-printer set up (A). Macroscopic images of fabricated 3D-printed scaffold immediately after printing (B), and after crosslinking (C, D). As can be seen in the images, the scaffolds are bendable with easy-handling properties. 


\section{Results and Discussion}

Printing Criteria: To describe the difficulties in finding the optimal gelatin printing parameters, rheological analysis has been performed.

Fig 1. presents the macroscopic images of the fabricated gelatin 3D-printed scaffold. To print successfully, 1) the temperature of the cartridge where the ink is kept, 2) the platform and 3) the dispensing needle are all of great importance. While the first two points can be adjusted precisely, the latter is dictated by heat transfer principles. The temperature of the cartridge must be set above the sol-gel transition temperature and the temperature of the platform needs to be set below this temperature.

The gel point of the ink was determined using oscillatory sweep measurements. The gelation temperature can be defined by observation of a frequency-independent value of damping factor $(\tan \delta)$ obtained from a multi-frequency plot for a temperature sweep measurement [11]. The solgel transition temperature of gelatin solution was found to be between $27^{\circ} \mathrm{C}$ and $29^{\circ} \mathrm{C}$ (Fig. 2A). Therefore, the cartridge and platform temperatures needed to be above $29^{\circ} \mathrm{C}$ and below $27^{\circ} \mathrm{C}$, respectively.

The shear stress and shear viscosity of the ink as a function of shear rate is demonstrated in Fig. $2 \mathrm{~B}$ and $2 \mathrm{C}$ for different temperatures. The high shear stress and viscosity at $19-28^{\circ} \mathrm{C}$ was because the measurements were conducted below the gel point. At these temperatures, the ink showed a shear thinning behavior as a result of disruption in physical network formed by hydrogen bonding between helices. It may come to mind that this apparent flow could be an artefact of delamination of the gel from the rotating plate. This assumption could be declined through considering oscillation 
data where complex viscosity vs. angular frequency shows the same trend (Fig. 2D). It should be noted that the shear stress, and consequently the pressure required to create a certain deformation and subsequently flow, is extremely higher when the ink temperature is below the gel point. As can be seen in Fig. 2, viscosity could be decreased by increasing the temperature from $31^{\circ} \mathrm{C}$ to $40^{\circ} \mathrm{C}$. The ink indicated Newtonian behavior (coefficient of determination, $\mathrm{R}^{2}$, greater than 0.99 ) at temperatures higher than $28{ }^{\circ} \mathrm{C}$. Fig. $2 \mathrm{E}$ displays the flow rate-pressure diagram for two standard dispensing needles at $37^{\circ} \mathrm{C}$. The relationship between the flow rate and pressure was nearly linear (coefficient of determination, $\mathrm{R}^{2}$, greater than 0.95 ) for pressures between 0 and 1.5 bar and two dispensing needles of 250 and $400 \mu \mathrm{m}$ in diameter. Since the ink represents Newtonian behavior at $37^{\circ} \mathrm{C}$, the Hagen- pressure and the flow rate are related by: $Q=\frac{\pi R^{4} \Delta P}{8 \mu l}$, in which $\mathrm{Q}$ is the flow rate, $\mathrm{R}$ is the radius of the pipe, $\mathrm{P}$ is pressure, $\mu$ is dynamic viscosity and 1 is the length of the pipe. The linear relationship between the pressure and flow rate, as well as the ratio of the slopes of the lines $\left(\left(\mathrm{R}_{2} / \mathrm{R}_{1}\right)^{4}, \mathrm{R}_{2}=400 \mu \mathrm{m}, \mathrm{R}_{1}=250 \mu \mathrm{m}\right)$, suggests that the flow of the ink through the needle followed the Hagen-Poiseuille's equation.

As can be seen in Fig. 2C, the viscosity remained almost constant for the temperatures between $34-40{ }^{\circ} \mathrm{C}$. It comes to mind that there should be no significant difference when any temperature in this range is selected. One should note, however, that the dispensing needle temperature is dictated by the balance between cartridge, room and platform temperature. When the printer is in standby mode (i.e. not printing and no material flow), the temperature of the remaining material in the dispensing needle starts to decrease and eventually drops below the gel point because of contact with room temperature. This results in obstruction of the dispensing needle and interruption of printing. However, this phenomenon does not occur instantaneously and there is a certain lag time between the standby and the onset of obstruction. This period is referred to as the "the golden time" 
in this article. To investigate the effect of cartridge temperature (i.e. the initial temperature of the material in the dispensing needle), as well as the effect of dispensing needle diameter on the golden time, rheological assessment with a plate-plate geometry was employed. The ink was loaded between the plates with various gap distances $(0.2-0.5 \mathrm{~mm})$ at different temperatures ranging from $37^{\circ} \mathrm{C}$ to $31^{\circ} \mathrm{C}$. The maximum cooling rate of the instrument was used to cool down the ink to $22^{\circ} \mathrm{C}$ while measuring the complex viscosity as a function of time in oscillatory mode at shear strain of $1 \%$ and frequency of $5 \mathrm{~Hz}$. The results are shown in Fig. 2F-I. Each gap distance is expected to resemble a certain needle diameter; if contact surface to volume ratio $(\mathrm{A} / \mathrm{V})$ is taken into the account, the gap distance of $0.2 \mathrm{~mm}$ resembles the blue needle $(\mathrm{D}=400 \mu \mathrm{m})$ characteristics $(\mathrm{A} / \mathrm{V}$ $\left.=10 \mathrm{~mm}^{-1}\right)$. However, this ratio is relatively big for the red needle $\left(\mathrm{D}=250 \mu \mathrm{m}, \mathrm{A} / \mathrm{V}=16 \mathrm{~mm}^{-1}\right)$ making it difficult to run the experiment with the resembled gap distance. By taking the time required to reach the target temperature by the rheometer into consideration, the period in which the complex viscosity remained unchanged analogizes the golden time though not precisely equal to it and the time at which the complex viscosity starts to ascend resembles the onset of needle obstruction. The delay of the rheometer was found to be in the range of 20-30 s as shown in Fig. 2F-I. Furthermore, the temperature of the rheometer platform is not necessarily equal to the material temperature as there is also a lag time to reach the equilibrium temperature applied by the rheometer. Such limitations cause the golden time measured using the rheometer to be longer than its actual value. As can be seen in Fig.2, the golden time was found to around 40, 35, 25 and $20 \mathrm{~s}$ for cartridge temperatures of $37,35,33,31^{\circ} \mathrm{C}$, respectively. Increasing the temperature from $31^{\circ} \mathrm{C}$ to $37^{\circ} \mathrm{C}$ can approximately double the golden time. On the other hand, the needle diameter does not seem to have had a significant effect on the golden time or the onset of obstruction at least up to $\mathrm{A} / \mathrm{V}=10$ 
but it did influence the speed of viscosity increase; the larger the gap (diameter), the slower the complete obstruction. 

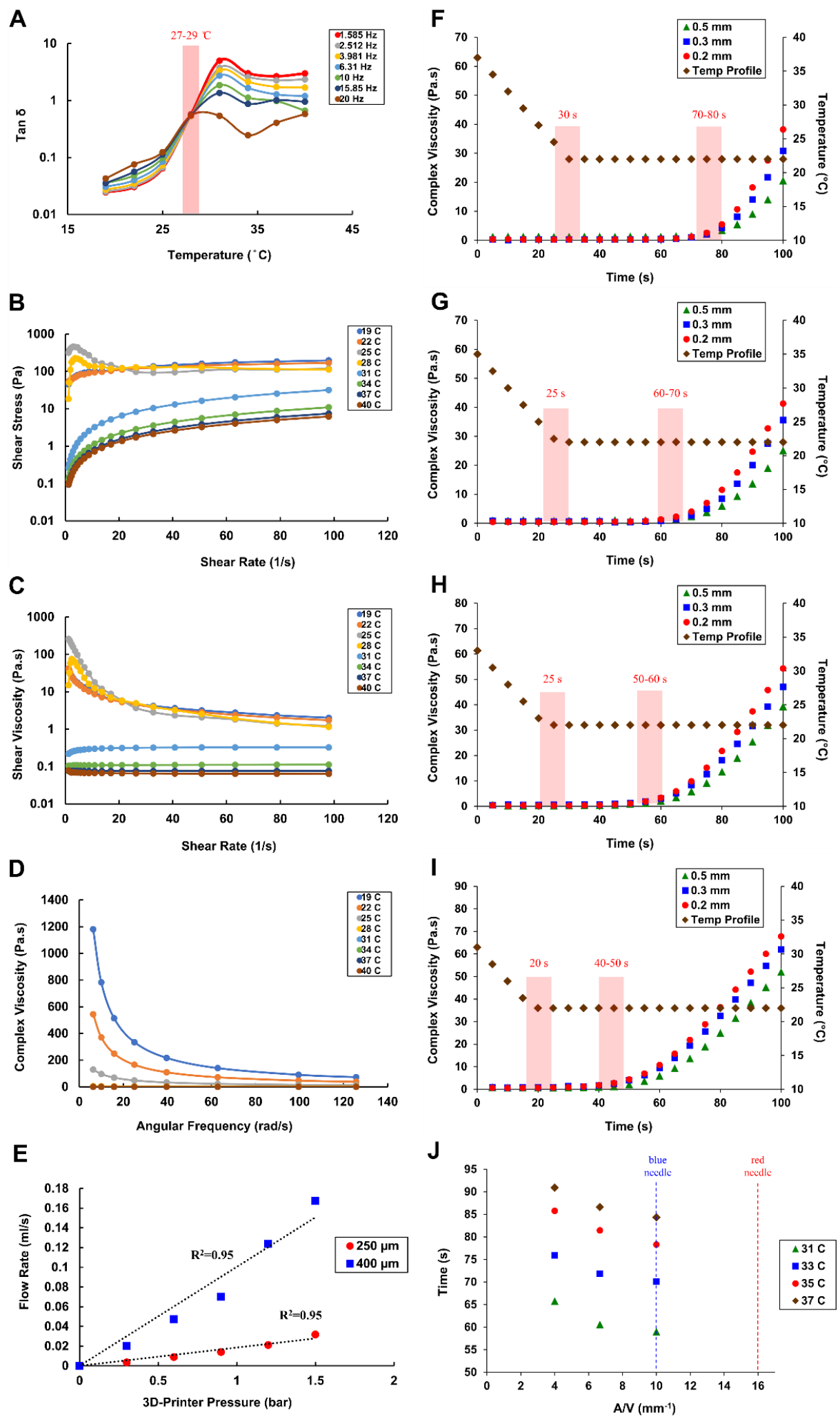

Figure 2: Rheological and flow characterization of Gelatin $15 \%(\mathrm{w} / \mathrm{v})$ aqueous solution as the ink for printing; sol-gel transition detection through oscillatory sweep measurements (A); shear 
stress and shear viscosity as a function of shear rate $(\mathrm{B}, \mathrm{C})$; complex viscosity as a function of angular frequency (D); flow rate as a function of applied pressure (E); complex viscosity change over time when cooling the solution from $37{ }^{\circ} \mathrm{C}(\mathrm{F}), 35^{\circ} \mathrm{C}(\mathrm{G}), 33{ }^{\circ} \mathrm{C}(\mathrm{H})$ or $31{ }^{\circ} \mathrm{C}(\mathrm{I})$ to $22{ }^{\circ} \mathrm{C}$ with maximum cooling rate; and the required time for the complex viscosity of the ink to reach 10 Pa.s as a function of surface to volume ratio $(\mathrm{J})$.

Fig. 2J represents the length of time required to reach the complex viscosity of $10 \mathrm{~Pa}$.s (including the delay time of the rheometer) as a function of contact surface to volume ratio (A/V). The significance of the effect of cartridge temperature compared to the contact surface to volume ratio on the golden time is again perceptible.

With regard to the effect of temperature on viscosity and golden time, $37^{\circ} \mathrm{C}$ was selected as the cartridge temperature. A $250 \mu \mathrm{m}$ needle was utilized since it was assumed not to have a significant effect on the golden time, it regulated flow rate variations caused by pressure fluctuation and yielded a better resolution because of lower strand thickness. The platform temperature was set at $22^{\circ} \mathrm{C}$ which was well below the gel point and very close to room temperature to avoid accelerated needle obstruction upon contact. The platform temperature affects the gelation time which in turn may influence the shape fidelity of the strands. Further investigation is required to optimize the platform temperature so that the shape of the strands is kept flawlessly while the printing is not disrupted because of needle obstruction.

To print an appropriate scaffold for oral mucosa as an example of heterogeneous tissue, the design criteria were as follows:

1. An appropriate thickness for oral mucosa scaffold ranges from $0.2 \mathrm{~mm}$ to $2 \mathrm{~mm}$.

2. In a novel model in oral mucosa scaffold designs, we printed the membrane in gradient shape with a large pore size on one side for the growth and penetration of connective tissue (i.e. fibroblasts) and a small pore size on the other side to accommodate keratinocytes (i.e. the epithelial 
layer). It is worth mentioning that the membrane in this study has been designed for post-fabrication cell seeding. If cell encapsulation is desired, gelatin methacrylamide or enzymatic protein crosslinking can be used.

3. The keratinocytes side needed to be permeable (i.e. no zero pore size) to allow nutrient diffusion and avoid reduced epithelial growth and differentiation.

Considering the above criteria and using the presented protocol, we printed our main scaffold by the following configurations: Using a $250 \mu \mathrm{m}$-diameter needle, six layers were printed while the distance between strands at each layer was set to approximately $300 \mu \mathrm{m}, 300 \mu \mathrm{m}, 450 \mu \mathrm{m}, 450 \mu \mathrm{m}$, $600 \mu \mathrm{m}$ and $600 \mu \mathrm{m}$ from the bottom to the top to achieve a gradient in pore size. The first and last layers' pore sizes are approximately 100 and $400 \mu \mathrm{m}$ (see Fig. 3A-B). The angles of strand-printing were selected to be $0^{\circ}$ and $90^{\circ}$ degrees on every other layer. The thickness of this membrane was initially $500 \mu \mathrm{m}$ and after cross-linking it shrunk to $300 \mu \mathrm{m}$. Such a low thickness might be a result of possible shape infidelity, stretching of the strand because of high printing speed and water evaporation from the sample. 

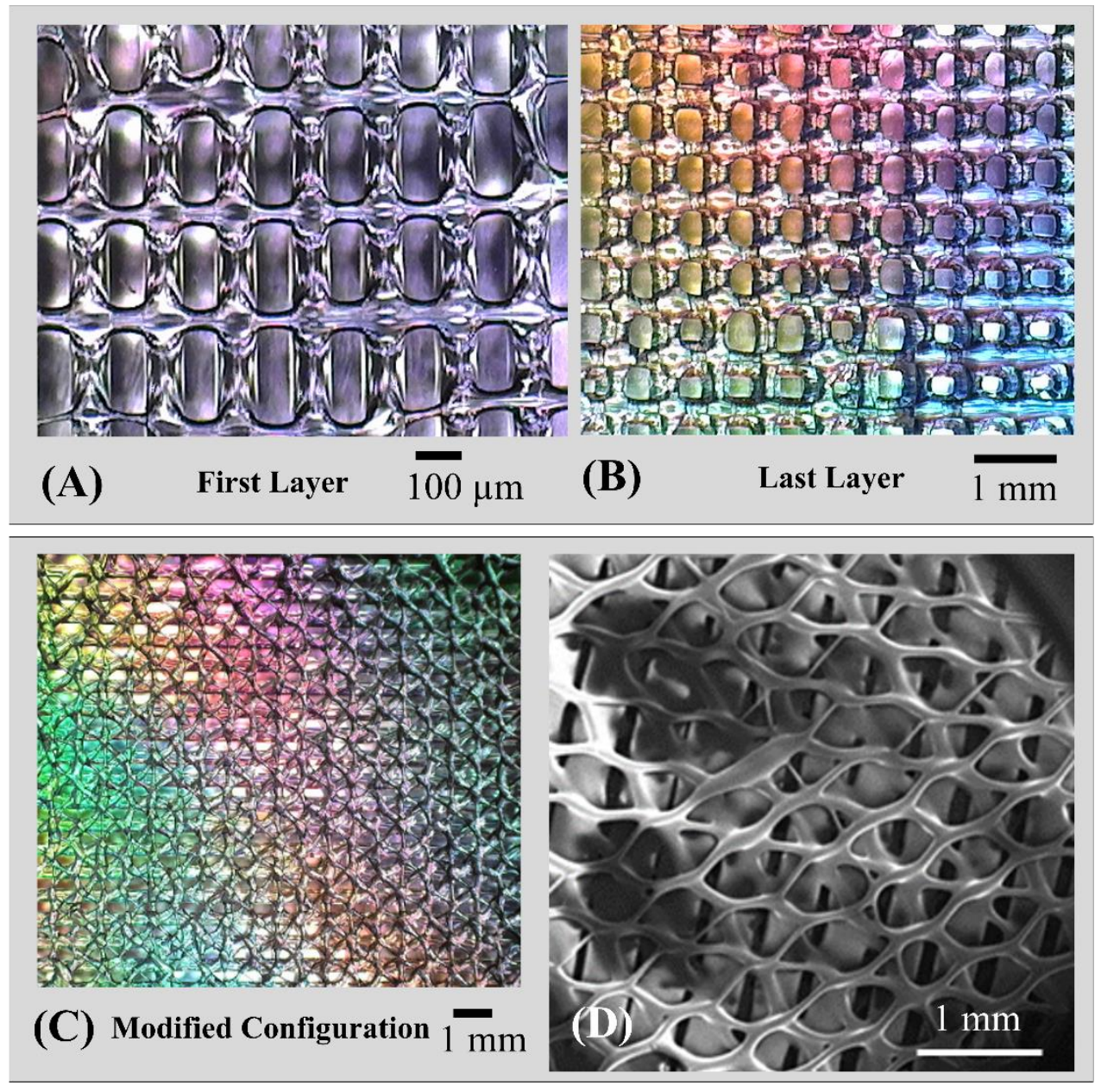

Figure 3: Examples of the first and last layers of the gradient membrane are shown in panel (A) and (B). The smaller pore size can be easily achieved by altering the angels of strands in the printing process (C). Panel (D) shows an SEM image of the printed membrane.

If required, smaller pore size on the epithelial side of the scaffolds can be achieved by changing the angle of the strand-printing on the last two layers to $45^{\circ}$ and $135^{\circ}$ instead of $0^{\circ}$ and $90^{\circ}$ (see Fig. 3C). The pore size can be reduced significantly by changing the angle of strands in various combinations. Fig. 3D demonstrates the SEM images of the membrane.

\section{Conclusion}

Employing a 3D-printing technique and using gelatin as the ink, we have introduced a novel scaffold design with potential application for tissue engineering of heterogeneous membrane-shape 
organs. The detailed printing process for gelatin, after consideration of its rheological characteristics is presented in this report.

As a particular example, the design criteria described in this paper have been used to produce a membrane that could be potentially used for oral mucosa tissue engineering.

\section{Acknowledgment:}

This work was supported by China Regenerative Medicine International Limited (CRMI).

\section{References:}

[1] S.F. Collins, Bioprinting Is Changing Regenerative Medicine Forever, Stem cells and development 23(S1) (2014) 79-82.

[2] R.S. B Starly 3D Bioprinting and Nanotechnology in Tissue Engineering and Regenerative Medicine. , in: J.F. L Zhang, K Leong, MA Waltham (Ed.), Three-dimensional (3D) Bioprinting, Academic Press2015.

[3] S.V. Murphy, A. Atala, 3D bioprinting of tissues and organs, Nature biotechnology 32(8) (2014) 773785.

[4] M. Guvendiren, J. Molde, R.M. Soares, J. Kohn, Designing biomaterials for 3D printing, ACS biomaterials science \& engineering 2(10) (2016) 1679-1693.

[5] B. Sarker, R. Singh, R. Silva, J.A. Roether, J. Kaschta, R. Detsch, D.W. Schubert, I. Cicha, A.R. Boccaccini, Evaluation of fibroblasts adhesion and proliferation on alginate-gelatin crosslinked hydrogel, PLoS One 9(9) (2014) e107952.

[6] T. Billiet, E. Gevaert, T. De Schryver, M. Cornelissen, P. Dubruel, The 3D printing of gelatin methacrylamide cell-laden tissue-engineered constructs with high cell viability, Biomaterials 35(1) (2014) 49-62.

[7] S.A. Irvine, A. Agrawal, B.H. Lee, H.Y. Chua, K.Y. Low, B.C. Lau, M. Machluf, S. Venkatraman, Printing cell-laden gelatin constructs by free-form fabrication and enzymatic protein crosslinking, Biomedical microdevices 17(1) (2015) 16.

[8] B. Duan, L.A. Hockaday, K.H. Kang, J.T. Butcher, 3D bioprinting of heterogeneous aortic valve conduits with alginate/gelatin hydrogels, Journal of biomedical materials research Part A 101(5) (2013) $1255-1264$.

[9] X. Wang, Y. Yan, Y. Pan, Z. Xiong, H. Liu, J. Cheng, F. Liu, F. Lin, R. Wu, R. Zhang, Generation of three-dimensional hepatocyte/gelatin structures with rapid prototyping system, Tissue engineering 12(1) (2006) 83-90.

[10] Z. Wu, X. Su, Y. Xu, B. Kong, W. Sun, S. Mi, Bioprinting three-dimensional cell-laden tissue constructs with controllable degradation, Scientific reports 6 (2016).

[11] H. Winter, Can the gel point of a cross-linking polymer be detected by the $\mathrm{G}^{\prime}-\mathrm{G}$ "crossover?, Polymer Engineering \& Science 27(22) (1987) 1698-1702. 